\title{
Perspectivas epistémicas para la generación de conocimiento en investigación educativa
}

\section{Epistemic perspectives for the generation of knowledge in educational research}

\author{
Nancy Barrios \\ Universidad Especialidades Espíritu Santo, Ecuador \\ Autor para correspondencia: nbarrios@uees.edu.ec \\ Fecha de recepción: 03 de Agosto de 2017 - Fecha de aceptación: 10 de Agosto de 2017
}

Resumen: La postmodernidad trajo consigo una serie de transformaciones en la sociedad, y la investigación forma parte de los mismos. De allí que, en la actualidad se asiste a una nueva racionalidad científica, las distintas posiciones epistemológicas abren caminos inusitados y la posibilidad histórica de plantear y replantear las cosas. El propósito de esta investigación fue Interpretar la concepción de la función de la investigación que tienen los docentes de la Universidad Pedagógica Experimental Libertador núcleo Maracay, Edo. Aragua, Venezuela. El enfoque fue cualitativo y el método empleado el hermenéutico. Se utilizó la técnica de la entrevista, los informantes estuvieron conformados por cinco (5) docentes del área de investigación. La interpretación de la información se realizó mediante las técnicas de categorización, estructuración, teorización y la triangulación. Entre los hallazgos se menciona que los informantes clave señalan que la investigación es un proceso sistemático de lo conocido y desconocido, señalan además que la estimulación a la investigación se da a través de las diferentes especialidades, asimismo expresaron que el positivismo es difícil de superar y que aún está en el proceso de investigación educativa, de igual manera manifestaron que la complejidad es un método y un paradigma. Por otra parte, señalan que la función de la investigación debe estar a la vanguardia para dar respuestas verdaderas a la comunidad al cual pertenece.

Palabras Clave: universidad; investigación; investigación educativa; paradigma; enfoque paradigmático

\begin{abstract}
Postmodernism brought with it a series of transformations in society, and the research is part of the same. From there, today we are witnessing a new scientific rationality, the various epistemological positions open unused roads and the historic opportunity of pose and rethink things. The purpose of this research was to interpret the role of the University in the research function at the Pedagogical Institute of Maracay Aragua Venezuela. The approach was qualitative and the method used the hermeneutic. We used the technique of the interview, respondents were comprised of five (5) teachers in the area of research. The interpretation of the information was done through categorization, structuring, theorizing and the triangulation technique. Among the finds is mentioned that key informants point out that research is a systematic process of what is known and unknown, likewise point to research stimulation is delivered through different specialties, also expressed that positivism is hard to beat and that is still in the process of educational research, in the same way demonstrated that the complexity is a method and a paradigm. On the other hand, they point out that research function should be at the forefront to give true answers to the community to which he belongs.
\end{abstract}

Key words: university; research; educational research; paradigm; paradigm approach 


\section{Introducción}

El mundo actual se caracteriza por un profuso proceso de desarrollo por ende, el hecho educativo no escapa de este avance de la sociedad. A tal efecto, la educación transita en este devenir de cambios en los diferentes órdenes que conforman el quehacer y devenir de la humanidad. A tal efecto, corresponde a la Universidad como agente protagonista de las vertiginosas transformaciones propias del siglo XXI, responder de manera vanguardista a esta evolución que se gestan en su entorno.

La educación como base ineludible para el desarrollo de la sociedad, a través de la preparación del individuo, debe responder a las exigencias del entorno, y de las circunstancias en las cuales le corresponda desempeñarse. Para ello, debe dirigir su acción al desarrollo de habilidades de una manera clara y con herramientas que le permiten al individuo conocer su misión como ser de la sociedad, amén de solucionar los problemas de la realidad en la cual le corresponde actuar. A este particular, la investigación viene a constituir la herramienta dentro de las ciencias sociales, para estudiar lo real y lo complejo de la humanidad, Así la Universidad desde esta función, emerge como el eje que direcciona la inquietud del quehacer educativo en pro de la solución de problemas. Hoy hay conciencia según Dupla (1999) más que nunca ha sido tan importante el conocimiento para la supervivencia del hombre como en los tiempos actuales.

Ello nos lleva a la búsqueda desde la universidad de nuevas coordenadas epistemológicas y ontológicas en sus procesos investigativos, que fundamenten una forma distinta de pensar y hacer ciencia y una manera diferente de formar al ser humano que se le entrega mediante la conformación de redes de intercambios académicos, tecnológicos y de investigación ya que, en este reto, la transcomplejidad constituye un par dialógico-recursivo valioso para la deconstrucción de las parcelas disciplinarias que han cercado y puesto límites al conocimiento y donde la cotidianidad surge como generadora de una racionalidad diferente que permite la construcción de relaciones sociales a través de la práctica social.

En la actualidad se asiste a una nueva racionalidad científica, las distintas posiciones epistemológicas abren caminos inusitados y la posibilidad histórica de plantear y replantear las cosas. En este marco, los estudiantes y docentes en la universidad tienen grandes posibilidades de manejar un cúmulo de métodos y tendencias que le permiten el abordaje de los fenómenos y hechos de su interés, desde múltiples perspectivas, tornándose, el proceso investigativo, más creativo, atractivo y libre de dogmatismos metodológicos.

En este sentido, desde la universidad se hace necesario un cambio en la concepción de la Investigación, ya que un proceso investigativo construido y ejecutado solo para fines academicistas, impide el surgimiento de redes de conocimientos, toda vez que dificulta el cumplimiento del rol protagónico como creadora, y gestora de conocimientos que tiene la universidad, lo que dificulta el pensamiento reflexivo y dialéctico, así como el aporte a las diversas disciplinas del conocimiento.

En el Instituto Pedagógico de Maracay, Estado Aragua (IPMAR), específicamente, en el área de investigación del Departamento Componente Docente (CODO), se congregan un grupo de docentes que tienen la responsabilidad de desarrollar las unidades curriculares de: 
Introducción a la Investigación e Investigación Educativa. Estas unidades constituyen el eje de investigación fundamental en el proceso de formación docente, por consiguiente, el discurso que maneje el profesor de estas asignaturas, de igual manera el telos que tiene la investigación para estos profesionales es determinante en la concepción y visión del proceso de investigación científica. Es así como se realiza el presente estudio, el cual persigue interpretar las concepciones epistemológicas de los docentes en el IPMAR, y a su vez conocer la importancia que la investigación tiene para el profesional de la docencia de este instituto de Educación Superior. De manera tal que a continuación se presentan los objetivos de la investigación;

\section{Propósitos de la Investigación}

1. Profundizar en la lógica discursiva del constructo Investigación Universitaria.

2. Interpretar la concepción de la función de la investigación que tienen los docentes del IPMAR.

3. Develar las expresiones más significativas que en materia de investigación educativa están presentes en el discurso del docente de investigación de CODO en el IPMAR

\section{Importancia}

Esta investigación aporta indicios sobre las diversas maneras de abordar el hecho educativo desde el contexto de la función de investigación en la universidad asimismo, pretende develar las diferentes visiones que poseen y practican los docentes universitarios al momento de indicar formas y maneras de abordar la realidad circundante desde una perspectiva problematizadora. Visto así, la relevancia de este estudio se enmarca en la posibilidad de aproximarnos a la diversidad de ideas, concepciones, limitaciones, aciertos y desaciertos si los hubiere, en la planificación, ejecución, control y evaluación de la actividad educativa de una realidad educativa en estudio.

Finalmente, desde el punto de investigativo como tal, la investigación se considera como un aporte inicial para futuras investigaciones que se interesen por conocer y ampliar horizontes sobre la pedagogía investigativa, el discurso paradigmático, el pensamiento por y para conocer, así como las importancia de la función de la investigación en el argot de la educación superior, entre otros.

\section{Referentes Teóricos}

El marco referencial constituye las bases sobre las cuales se sustenta la investigación, en él se exponen los conceptos, constructos y categorías. Al respecto Tedesco (1999), afirma que: Representa el soporte inicial de la investigación (...) es el constructo teórico en el que se apoya la misma... aborda las características del fenómeno o problema en estudio. Estas características (cualidades) constituyen las variables que no son más que las diferentes proposiciones, diversos conocimientos, que permitirán estudiar el problema. (p.51). Es necesario destacar que debido a que este estudio se realiza desde la perspectiva metodológica cualitativa, el aspecto teórico solo corresponderá a un referente, y no a una posición teórica.

\section{Antecedentes de la Investigación}

Esteves (2014) en su Trabajo de Ascenso para la categoría de titular en la Universidad de los Andes intitulado, "El Rol de Investigación del Docentes en el Marco de la Postmodernidad". 
La investigación fue desarrollada bajo la modalidad de campo y documental, utilizando el método crítico reflexivo. Entre los aspectos que destaca el autor en su investigación se encuentra que el proceso a través del cual una organización llega a ser de modo diferente de lo que era en un momento dado anterior es mediante al cambio. Además expresa que, todas las organizaciones cambian pero el reto que se plantean los docentes $\mathrm{y}$, en general, todas las personas de la organización es que el cambio organizacional se produzca en la dirección que interesa a los objetivos de la organización. Es por ello que se habla de la investigación como factor de transformación y cambio. En los resultados se pudo evidenciar a través de sus resultados, que los docentes carecen de la capacitación mínima en relación al compromiso que como investigadores tienen. Asimismo, observó que el 75\% de los encuestados no tienen el conocimiento para realizar investigación. Al 84\% de los docentes participantes en la investigación también opinaron que los entes gubernamentales no los capacitaban al respecto, y que por otra parte, no se pueden ausentar de la institución porque las autoridades se lo impiden. Finalmente, la autora de esta investigación presentó en sus conclusiones que el rol investigativo de los docentes se expresa a través de los Proyectos de Aula y algunos de ellos aún tienen sus dudas al respecto.

Bermúdez (2015), realiza una tesis doctoral titulada, Teoría Epistemología Humanista $Y$ Científica De La Praxis De La Investigación Educativa A Través De La Sistematización De Experiencias. En el estudio, se plantea construir un aporte teórico, a partir de la experiencia de vida de los pensadores e investigadores educativos sobre los nuevos significados epistemológicos que sirven para orientar lo que serán los nuevos senderos por donde deberá transitar la praxis investigación científica, que conlleven a la reconfiguración de su significado humano y científico. Por otra parte, se desarrolló atendiendo al enfoque cualitativo y método hermenéutico, emergiendo el método de la sistematización de las experiencias, donde sus planteamientos gnoseológicos confirman el carácter interpretativo de la investigación, ya que la realidad es conocida desde la perspectiva y experiencia de los actores involucrados en el evento de estudio. Para el proceso de abstracción de la teoría que emergió de la descripción de las experiencias de la vida se seleccionaron cuatros informantes sustantivo, los cuales fueron escogidos de tres universidades del país. La teoría de primer nivel (síntesis Integrativa), emergente, de la sistematización de las experiencias, plantea un cambio de dirección, una nueva mirada, una vuelta a las fuentes originarias de la investigación científica; es decir, una investigación nueva, desnuda, más humana, cercana y simple, irreductible a los protocolos metodológicos e intereses ajenos a la naturaleza onto-antropo-epistemológica de las realidades objeto del pensamiento e investigación científica.

\section{Constructos Teóricos}

\section{Educación Universitaria e Investigación}

La situación de la investigación en pregrado no ha sido motivo de búsqueda y debate sólo en Venezuela; sino en otras latitudes investigativas también se han preocupado por este aspecto. En este sentido, Brunner (1998) señala que en América Latina más de la mitad de los investigadores están en las universidades y en ellas se produce una proporción significativa de las actividades locales de investigación y desarrollo. Por tanto, la universidad está llamada a ser uno de los polos de crecimiento en un momento histórico en la cual ya los recursos naturales de 
los países, por sí mismos, no basta para el desarrollo, y cada vez cobra mayor importancia la capacidad para generar, concentrar, asimilar y aplicar nuevos conocimientos.

La investigación universitaria constituye el nexo de un número de responsabilidad esenciales de la sociedad, entre ellas, mantener la infraestructura científica, desarrollar capacidades para explorar nuevos campos de la investigación, formar generaciones de científicos, proporcionar a la docencia la dimensión científica que requiere, conducir la investigación básica necesaria para la innovación, la generación y la difusión de conocimientos (Licha, 1992). En otras palabras, la universidad y los centros especializados en la formación de investigadores, en lo que respecta a la investigación está llamada a cumplir cuatro tareas sociales:

1. Fundamentar el sistema nacional de investigación.

2. Contribuir a la formación de científicos.

3. Contribuir al cambio técnico.

4. Contribuir al cambio del conocimiento.

Según el artículo 27 de la Ley Orgánica de Educación en Venezuela (2009), uno de los objetivos de la educación superior es fomentar la investigación de nuevos conocimientos e impulsar el progreso de la ciencia, la tecnología, las letras, las artes y demás manifestaciones creadoras del espíritu en beneficio del bienestar del ser humano, de la sociedad y del desarrollo independiente de la nación.

\section{Profesor Universitario y sus Competencias en la Investigación}

El dominio del saber científico por parte de una sociedad constituye en el mundo contemporáneo, una condición básica para afirmar la identidad y la independencia cultural de cada país, para favorecer la participación de la población en la ejecución de acciones hacia el desarrollo y para permitir un mejor control nacional de los limites (UNESCO, 1990).

Este proceso evidencia que el desarrollo de la sociedad y del ser humano se subordina cada día más a la capacidad de los pueblos para crear, innovar, manejar información y conocimientos, investigar y aplicar los inventos y descubrimientos que la investigación produce, de modo que la riqueza y bienestar de las naciones estarán condicionados ya no solo por los recursos naturales o materiales que posean, sino por sus recursos y potenciales intelectuales. (Guadilla, en Hurtado y Toro, 2000)

Según el planteamiento de numerosos autores, entre los que se encuentra Albornoz (2007), el perfil de la ciencia en América Latina, puede sintetizarse de la siguiente manera:

1. Escaso tamaño de comunidad dedicada a tareas de investigación y desarrollo.

2. Marcada debilidad política de los gobiernos para otorgarle prioridad a la inversión en investigación.

3. Escasa participación del sector productivo el financiamiento de actividades de investigación y desarrollo.

4. Escaso contribución de la producción científica Latinoamericana en relación al total mundial.

5. Fuerte concentración de la capacidad científica y tecnología de la región en tres países: México, Brasil y Argentina. 
6. Redes de generación y transmisión de conocimientos tenues, y escaso aprovechamiento de estas por la economía y la política.

7. Limitada efectividad para transformar la realidad.

8. Escasa valoración social del conocimiento.

9. Fuga permanente de talento.

10. Ausencia de cálculos acerca de los costos y la productividad de las investigaciones que se hacen en la región.

En Venezuela la problemática anteriormente expuesta, disímil, presenta una situación que se ha prolongado en el tiempo a pesar de los múltiples esfuerzos y reflexiones que se han hecho en torno a esta circunstancia. Por lo que de acuerdo con el autor antes mencionado, para inicios del 2000, Venezuela tiene un déficit de por lo menos 15.000 investigadores para cumplir los requerimientos compatibles con un desarrollo científico tecnológico satisfactorio. No por ello debe asumir posiciones conformistas, al contrario, desde las Universidades, debemos fortalecer la investigación y de inmediato ha de atenderse como un aspecto prioritario la producción de conocimientos así como la formación de investigadores en el contexto de la sociedad del siglo XXI. Por lo tanto, la universidad está llamada a ser uno de los polos de crecimiento en un momento histórico en cada vez cobra mayor importancia el generar y aplicar nuevos conocimiento.

\section{Perspectiva Epistémica}

Todo proceso de investigación tiene lugar en el contexto de las experiencias personales de quien las realiza, en un conjunto de creencias científicas, unas normas socioculturales generales y de ciertas tradiciones filosóficas, todo lo cual contiene supuestos acerca de la naturaleza de la realidad, del conocimiento y de los valores, que interactúan con los constructos de la ciencia en que el investigador enmarca su trabajo.

Es por ello que, los métodos de investigación y las opciones metodológicas son parte integral de los sistemas teóricos empleados por el investigador .En la búsqueda del conocimiento, el modelo conceptual que se adopte va a condicionar los procedimientos de estudios que se sigan en la investigación. En este sentido, cobra vital importancia el enfoque paradigmático que se asuma. De acuerdo a Pérez (2004) es evidente que cada paradigma mantiene una concepción diferente de lo que es la investigación, lo que implica la adopción de metodologías determinadas, en otras palabras, los paradigmas definen el marco del pensamiento o referencias que orientan las actividades y las reflexiones dentro de un área determinada del saber.

El descontento y la intuición de que el paradigma científico tradicional, no responde a los nuevos retos que demanda este siglo dominado por una proceso globalizado que deconstruye las antiguas y clásicas corrientes filosóficas, ha llevado a la necesidad de plantearse nuevas reglas del pensar y nuevas formas de hacer conocimiento; lo que ha dado origen al surgimiento de un nuevo paradigma que debe elevarse sobre el pensamiento actual. De acuerdo con Morín (1999), la incapacidad del pensamiento actual para percibir y concebir lo global y fundamental, la complejidad de los problemas humanos; demanda un nuevo pensamiento que una lo que está separado y compartimentado, que respete lo diverso, multidimensional, ecologizado, que reconozca la incompletud, entre otros. Lo expuesto en párrafos anteriores, demanda a la 
investigación en el campo de la educación salidas oportunas que no pueden lograrse bajo una óptica determinista, mecanicista, y formal, la cual ignora, que los fenómenos estudiados están influenciados por metadimensiones, por los sentimientos del propio investigador, y por el hecho en sí, que otros actos ocurridos en otros lugares afectan el objeto de estudio. Es por ello, que se requiere de un nuevo paradigma que sea cónsono con los avances epistemológicos de las últimas décadas, consciente de que no existen modelos por encima, de otros ni lenguajes diferentes para explicar los acontecimientos y proteger al hombre de sí mismo, a través de un pensamiento de procesos e interrelaciones y no de forma aislada.

En tal sentido, las nuevas realidades han demandado el surgimiento de una nueva visión paradigmática, un paradigma emergente de la ciencia como lo denomina Martínez (2004), que permita desde una perspectiva más amplia holística y ecológica, describir el mundo actual, es decir, se requiere de una ciencia más universal e integradora. Por estas razones, este trabajo de ascenso se acoge a los postulados del paradigma Post positivista de la ciencia, el cual, desde la perspectiva del autor antes referido, debe "brotar de la dinámica y dialéctica histórica, de la vida humana y se impone, cada vez más con fuerza y poder convincente, a nuestra mente inquisitiva" (p.156). De hecho, la ciencia es consecuencia de la racionalidad e inteligencia humana y es éste el hombre el único capaz de cambiar sus propias interpretaciones sobre la realidad a fin de impulsar cambios en la ciencia moderna. La diferencia fundamental de orden epistemológico entre el positivismo y el post positivismo se deriva de su gnoseología. En estos últimos, se supera el esquema que considera la percepción como simple reflejo de las cosas "reales". En el conocimiento se da una interacción, una dialéctica entre el conocedor y el objeto conocido, por ellos no se pueden enfatizar la objetividad.

\section{Perspectiva Ontológica}

Algunos autores, entre ellos Sánchez (2008), comparten la idea de que el hombre es pura posibilidad; en este sentido, lo visualizan como poseedor del habla para pronunciar las cosas, para denominarlas y darles significados. Pero también el hombre es un ser carente, casi, limitado por la condición de estar situado entre factores socioeconómicos, culturales e históricos, y por ser objeto de controles, sociales normatizado por leyes y delimitados por códigos jurídicos. No obstante, para esta investigación el hombre se define como ser en el mundo, ser con otros y ser inconcluso. El hombre ser en el mundo significa que él está relacionado esencialmente con su teoría, un ser espacio temporal, individuo en continuar intervención con su medio.

\section{Perspectiva Gnoseológica}

El conocimiento es el resultado de la relación entre un sujeto cognoscente u el objeto por conocer. Desde el punto de vista epistemológico los abordajes empíricos y positivistas privilegian al objeto o al hecho, haciendo desaparecer al sujeto en pro del objeto, suponen que el objeto real y el objeto del conocimiento permanecen reducidos a la representación del primero sin permitir la interacción del sujeto, suponen también que la relación sujeto-objeto es una relación de duplo empirismo entre un individuo concreto y aislado (investigador) y un pedazo de realidad también concreta sin embargo, el sujeto empírico es portador de una cultura, de un lenguaje de una estructura de pensamiento y de una escala de valores su relación con el objeto en este sentido no es neutra. El sujeto y el objeto del conocimiento no son dos entidades que entran en reacción a través de una tercera entidad llamada sensación, observación o experimentación, sino que son dos aspectos de una misma realidad en anudad y contradicción dialéctica. 


\section{Perspectiva Axiológica}

Teniendo en cuenta las contribuciones de Habermas (1980) sobre la relación "conocimiento e interés", es necesario considerar algunas cuestiones relacionadas con las actitudes, posturas u compromisos del investigador durante los procesos de construcción del conocimiento sobre la educación, considerando que su problemática por el hecho de ser social, política e ideológica, no es neutra ni está libre de connotaciones de valor. Por el contrario, está saturada de elementos axiológicos, de concepciones filosóficas de hombre sociedad, visiones del mundo, que la transforman en un campo de interminables controversias y que exige de los profesionales una postura o una actitud comprometida, a favor o no de los valores y de las decisiones que están en juego en esa compleja problemática.

\section{Método de Investigación.}

El paradigma post positivista como enfoque, demanda de un método que vaya más allá del objetivismo y la determinación, para abordar la compleja realidad del conocimiento en el campo de la educación. En función de lo anterior se considera que la herramienta metodológica más apropiada para interpretar el fenómeno objeto de estudio es el método hermenéutico.

La hermenéutica es la disciplina de la interpretación, trata de comprender textos, en sus contextos respectivos, lo cual es dicho de manera muy amplia. Con eso el intérprete o investigador los entiende, los comprenden, frente a sus autores, sus contenidos y sus destinatarios, estos últimos tanto originales como efectivos. Esta disciplina se ve omnipresente y variopinta, de matices muy diferentes, muestra una cara múltiple pero procurando no traicionar la gran diversidad de planteamientos, tratará de reunir aquí algunos de sus rasgos, problemas y perspectivas más básicos, de modo que puedan servir de contacto inicial con ella., tiene sus orígenes históricos desde los griegos, Aristóteles, en su Peri hermeneias, dejo muchas ideas inapreciables sobre ella.

Según lo que describe Martínez (2004) acerca del método hermenéutico-dialéctico, en esta investigación se cubrieron varios momentos:

Primer Momento: incorporación de las investigadoras al medio natural de los sujetos de estudios; incluye el uso de todas las técnicas empleadas para recopilar la información (entrevista observaciones, anotaciones, grabaciones).

Segundo Momento: audición de la grabaciones, lecturas de las transcripciones, anotaciones al margen de la mismas, lectura de los registros de observación. Aquí se trata de pasar del dato a la vivencia misma de quien la produce, tal como dice Dilthey: "comprender significa pasar de la exterioridad de los datos a la vivencia originaria que los produce, sean ellos gestos, lenguaje u objetos culturales en general" (Moreno, 1995, p. 77)

Tercer Momento: categorización de la información con las categorías provisionales disponibles y con la categorías emergentes del propio material.

Cuarto Momento: estructuración de la información, mediante la cual se brinda una visión comprehensiva del protocolo correspondiente a cada una de las entrevistas efectuadas. 
Quinto Momento: Determinación de los quiebres o rupturas epistémicas.

Sexto Momento: teorización para integrar nuestras conclusiones y relacionarlas con el campo teórico respectivo existente.

\section{Técnicas e Instrumentos de Investigación}

\section{La entrevista en Profundidad}

La entrevista constituye una técnica que proporciona significativos resultados en el desarrollo de un estudio cualitativo. En esta investigación, las entrevistas se caracterizarán por su apertura, flexibilidad y dinamismo, este tipo de entrevistas son denominadas como entrevistas en profundidad de acuerdo a Taylor y Bodgan (1997).

\section{Instrumentos}

Para tal propósito, se precisó de una Guía de Entrevista, para asegurar que la diversidad de temas incluya los aspectos claves a explorar. Dichas entrevistas fueron grabadas y en algunos casos fueron registradas en el Diario de Campo, donde se transcribieron las conversaciones, añadiendo los atributos utilizados por el entrevistado referidos a situaciones, objetos o personas.

\section{Informantes Clave}

Según Martínez (2004) la selección de los informantes clave en una investigación cualitativa no admite la escogencia al azar, aleatoria y descontextualizada. De igual forma, de acuerdo a lo planteado por Rusque (2001), la selección de informantes clave es flexible y abierta hasta llegar a la saturación o redundancia teórica de las unidades de análisis, categorías y subcategorías que surjan.

En función de lo anterior, en el presente estudio la selección se realizó de manera intencional, válido para este tipo de investigación, donde se prioriza la profundidad acerca de la extensión y la muestra se reduce en su amplitud numérica. Los criterios utilizados para seleccionar los actores clave fueron: docentes del Pedagógico de Maracay. De igual manera se consideró que fueran docentes del área de Investigación del Pedagógico de Maracay

A tal efecto, a continuación se hace una breve descripción de los informantes que participaron en este estudio.

Informante 1; Hipotético Deductivo: Hombre de 47 años de edad, alto delgado, blanco con entradas profundas en la frente de carácter amable y es muy colaborador.

Informante 2; Etnografía. Mujer de 42 años de edad, blanca, de contextura gruesa, cabello negro de aspecto amigable y sonriente, dispuesta siempre a ayudar a sus compañeros.

Informante 3; Hermenéutica: Mujer de 42 años de edad, blanca de contextura delgada, de mediana estatura, cabello claro, amable en su trato, es colaboradora con sus compañeros. 
Informante 3; fenomenología: Mujer de 33 años de edad, blanca de estatura baja, delgada, cabello negro usa lentes, siempre está sonriente, es agradable en el trato.

Informante 5; Historia de Vida: Mujer de 53 años de edad, trigueña, alta, delgada cabello negro corto, dedica gran parte de su tiempo a la investigación.

\section{Escenario de la Investigación}

La presente investigación se desarrolló en el Instituto Pedagógico de Maracay, Estado Aragua. Venezuela

\section{Estrategias de Procesamiento de la Información}

El procesamiento de la información constituye una de las tareas más complejas dentro del proceso de investigación. Al respecto Martínez (2004) señala: Los datos recogidos en el campo, constituyen las piezas de un puzzle que el analista se encargará de ir encajando, utilizando la evidencia recogida para orientar la búsqueda de nuevas evidencias susceptibles de incorporarse a un sistema emergente de significados que da cuenta a la realidad estudiada y que poco a poco va aproximando al investigador a la descripción y la comprensión de la misma. (p. 197).

Pero los datos por sí solos, no son suficientes para obtener conclusiones en una investigación; la información constituye la materia prima que le servirá al investigador a través de las técnicas de análisis, para estructurar el conjunto de información en forma coherente y significativa en relación con el problema de estudio. A pesar de que las técnicas de análisis de información, en la mayoría de las ocasiones surgen durante el desarrollo de la investigación y son pautadas por el interpretador dentro del proceso de estudio, a continuación se describirán algunas de las técnicas que las investigadoras consideraron pertinentes en relación con los objetivos planteados:

a) Categorización: Permitió categorizar o clasificar la información según las unidades de análisis, las cuales fueron descritas y luego se diseñó una categoría de acuerdo a las clases significativas, a medida en que se revisó el material fueron emergiendo los significados de cada evento, hecho o dato. Posteriormente se realizó una síntesis analítica por cada categoría y se resumió a nivel de conclusión.

b) Triangulación: a través de los informantes clave, la teoría y las investigadoras, la cual permitió contrastar los diferentes puntos de vista que tienen los implicados acerca de una misma realidad. En tal sentido, se efectuó un análisis descriptivo e interpretativo de la información, cuya base es la Hermenéutica Dialéctica.

c) De cada estructura particular, se concentró en una estructura general formándolo así en un todo.

d) Partiendo de la estructura general se procedió a elaborar el discurso de la síntesis conceptual y las reflexiones finales.

Es precisamente ante esta posibilidad, que las investigadoras abren un nuevo camino, porque la interpretación mediante el método hermenéutico, permitió la reconstrucción del sentido de los significados construidos, que unido al proceso de teorización, fue posible develar las características de una realidad.

\section{Triangulación}


Según Taylor y Bogdgan (1990) la técnica de la triangulación consiste en "la combinación, un estudio único de los distintos métodos o fuentes de datos". Basado en ello, se realizó la triangulación combinando los contenidos de las entrevistas, los registros de observación y la teoría, con esto se logró comprensión clara y detallada de la situación presente en los distintos escenarios observados, así como en las opiniones y juicios emitidos por las personas estudiadas. De igual manera y para los mismos fines se consideraron los contenidos de todos los documentos pertinentes y fuentes de datos que pudieran ser manejados y analizados.

\section{Los Hallazgos}

A continuación se presentan los resultados obtenidos mediante la aplicación de la técnica entrevista semiestructurada y procesados a través de la categorización de los diferentes contenido protocolares y confirmada mediante las estructuras particulares, matriz de triangulación y estructura general.

\section{Estructura General:}

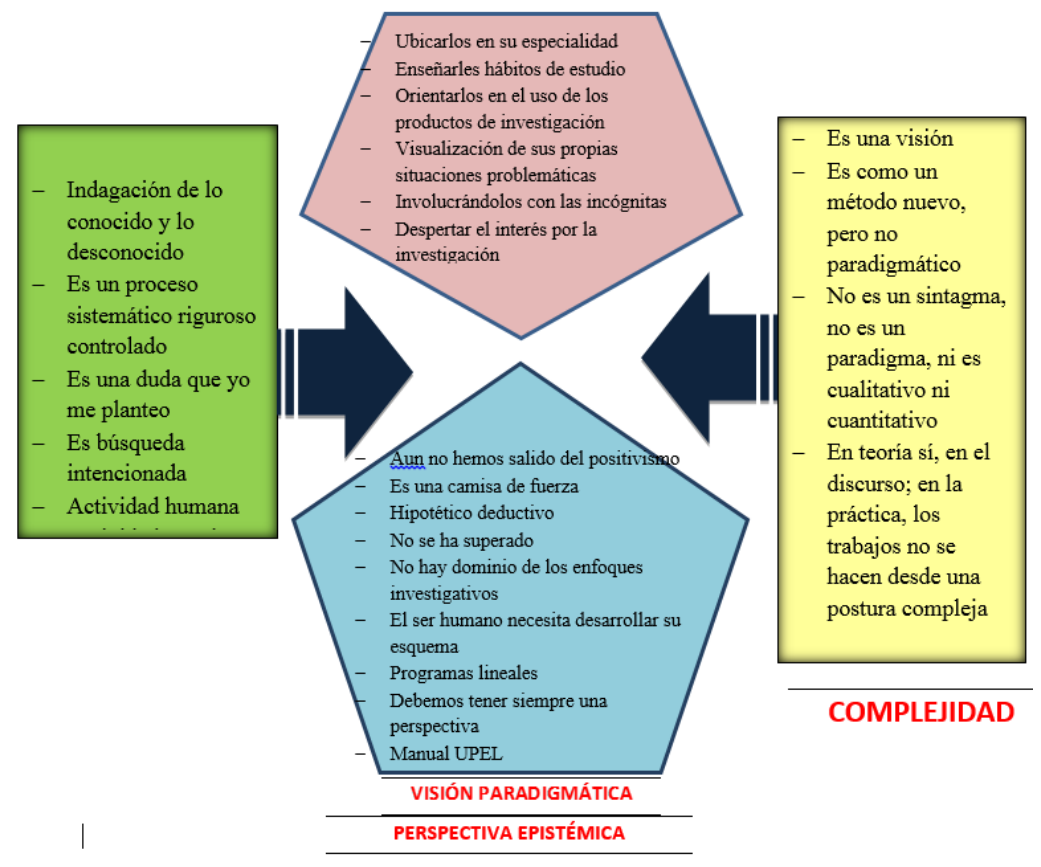

\section{Síntesis Conceptual}

\section{Teoría de Primer Nivel}

La investigación es una función fundamental de la universidad; constituye un elemento importantísimo en el proceso educativo porque a través de ella se genera conocimiento y se propicia el aprendizaje para la generación de nuevo conocimiento; además, la investigación vincula la universidad con la sociedad. Al ser una función fundamental, sustantiva, la investigación es un deber ser. Por esta razón, las universidades deben desarrollar capacidades para la investigación en los estudiantes e incorporar la investigación como estrategia de enseñanza y aprendizaje. En el mundo científico vivimos momentos telúricos y de gran incertidumbre, precisamente por una especie de crisis en los paradigmas heredados de la modernidad, cuyas rígidas estructuras metodológicas no permiten penetrar en los profundos 
parajes de un universo lleno de complejidad. Esta sacudida paradigmática está teniendo un serio impacto en lo epistemológico y en lo humano, que ha obligado a repensar las bases de la ciencia, sus estatutos y los enfoques que orientan la hechura del saber. Por eso hablamos de un movimiento paradigmático y con mucha razón, pues el Siglo XX, a nuestro modo de ver, construyó las bases teóricas mediante las cuales se conducirá la ciencia del futuro. Einsten con la teoría de la relatividad, Heisenberg con la teoría cuántica y de la incertidumbre y Prigogine con la teoría del caos, asestan un golpe mortal al discurso paradigmático de la modernidad, demoliendo los últimos ladrillos de la lógica reduccionista, objetivista y cuantitativa, al demostrar que el universo es más complejo que lo representado por modelos tautológicos y simplistas creados por el paradigma científico decimonónico.

No obstante, se aprecia en lo expresado por los informantes, una tendencia hacia el positivismo lógico, manifiesta a través del discurso en la constante mención a variables, análisis, esquemas, objeto, defensas, proyectos factibles, explicación y control. Todo ello constituye un claro indicio de que en el pensamiento de nuestros investigadores prevalece una clara tendencia paradigmática: el positivismo. Así se refleja en el pensamiento de los informantes una concepción de la realidad limitada por coordenadas de: tiempo, espacio y masa, puesto que solo considera la posibilidad de estudiar científicamente los hechos, los fenómenos, el dato experimentable, lo observable, lo verificable para lograr el progreso de la sociedad. Asimismo, se refleja una realidad donde los docentes de acuerdo a las respuestas emitidas a la entrevistas, no consideran la importancia que la investigación tiene para en contexto universitario, y en sí para la comunidad en general.

En consecuencia, en esta investigación los hallazgos apuntan a que el pensamiento de los docentes de investigación en el instituto Pedagógico Rafael escobar Lara, de Maracay, en el contexto del debate postmoderno, mantienen una concepción epistémica, en términos de Maturana, supuestamente crítico y post positivista, pero que en el fondo no logra superar ni epistemológicamente ni en el metodológico a los viejos esquemas positivistas. De manera que con nuevos ropajes seguimos tapando cuerpos enraizados en la vieja racionalidad científica.

Por último es importante subrayar las palabras de Zaa (2008), quien expone que, la investigación científica depende de la calidad de los investigadores. Estos no se improvisan y es necesario formarlos cuidadosamente. El cultivarlos es una tarea larga y difícil, más que la de obtener las plantas más delicadas y las flores más preciosas.

\section{Bibliografía}

Aguerrondo, I (1992). La Innovación educativa en América Latina: balance de cuatro décadas, en: Perspectivas, vol. XXII, No. 3, pp. 381-394.

Albornoz, Orlando (2007). Universidad y calidad: experiencias y proyectos. Primera edición, 2007. CUniversidad de Los Andes. Vicerrectorado Académico. Centro Editorial Litorama C. A.

Bermejo, B. (2003). Método Empírico de Recolección de Datos. Compendio de Metodología Cualitativa en la Educación. Sep-Dic. Candidus. 58-69 
Bermúdez, J. (2015). Teoría, Epistemología Humanista y Científica de la Praxis de la Investigación Educativa a través de la Sistematización de Experiencias. Trabajo Doctoral no publicado. Universidad Experimental Libertador (UPEL), Maracay Aragua, Venezuela

Buendía, L. (1998). Métodos de Investigación en Educación. Madrid: Autor.

Bunge, M. (1980). Epistemología. Barcelona - España: Ariel.

Blumer, H. (1969). Interaccionismo Simbolico: perspectiva y método. Nueva Jersey: Prentice Hall.

Consejo Nacional de Universidades (1993). Orientaciones y Lineamientos para la transformación y Modernización del Curriculum Universitario. Caracas:

Delgado F. (2001). Paradigmas y Retos de la Investigación Educativa. Una Aproximación Crítica. Mérida: Universidad de Los Andes.

Delors, J. (1997) La Educación encierra un tesoro. Informe a la UNESCO de la comisión internacional sobre la educación para el Siglo XXI.

Dilthey, W (1944) Ideas acerca de una psicología descriptiva y analítica, en Eugenio Imaz (editor), Obras (México: Fondo de Cultura Económica) Tomo VI, p. 196.

Dilthey, W. (1951) Historia de la filosofía. MX: Fondo de Cultura Económica. — 271 p.; Solicitar en: Bib. Fac. Derecho y Cs. Sociales 19-7-2 (09) D 14237

Duplá, F (2000) La Educación en Venezuela. Curso de formación Sociopolítica. № 37 Caracas: Gumilla.

Duplá, J. (1999). La Educación en Venezuela. Caracas: CERPE.

Estévez C. (2014). El Rol de la Investigador del docente en el Marco de la Post modernidad. Trabajo se Ascenso para titular no publicado. Universidad de los Andes. Venezuela.

Espinoza, I. (1997). Aproximación Teórica al Educador - Investigador. Caracas: Los Heraldos Negros. Fernández de Silva, I. (2000). Diccionario de Investigación Holística. Caracas: Sypal

Gadamer, H (1999). La Historicidad de la Comprensión como Principio Hermenéutico. Verdad y Método I. Ediciones Sigueme. España

Gibaja, R. (2002). Acerca del Debate Metodológico en la Investigación Educacional. En: La Educación, Revista Latinoamericana de Desarrollo Educativo., 32 (103), 81 - 83.

Guzmán (2006). Tras las huellas de Atenea. Nociones básicas y reflexiones sobre Epísteme y Paradigmas occidentales del conocimiento. IVISED. Caracas, Venezuela

Habermas, J. (1997). Ciencia y técnica como ideología. Ed. Tecnos., Madrid. 
Hernández, L. (2005). Una Vía Transdisciplinar Sobre Las NTIC Para el Desarrollo De Habilidades Profesionales Generales en Cursos de Postgrado Semipresenciales.Instituto Superior Politécnico José Antonio Echeverría. ISPJAE. Cuba.

Hurtado I y Toro J. (2000). Paradigmas y Métodos de Investigación en tiempos de Cambio. Episteme Consultores y Asociados. Valencia Venezuela.

Ley Orgánica De Educación (1999). Gaceta Oficial de la República de Venezuela, № 36.787. 15 de septiembre de 1999.

Lyotard, J. F. (1984). La Condición Postmoderna. Cátedra UNESCO

Marqués, P, (2000) Metodologías de Investigación. Modelo para el Diseño de una Investigación Educativa.

Martínez Miguélez, M. (2004). Ciencia y arte en la metodología cualitativa. México: Trillas.

Martínez, J (2004) La Nueva educación para la Sociedad del Conocimiento. Barcelona España: Ariel

Moreno, M (1995), Investigación e Innovación Educativa, Revista la Tarea No. 7, disponible en URL: http://www.latarea.com.mx/articu/articu7/bayardo7.htm

Morín, E (1999) Los siete saberes necesarios para la educación del futuro ICFES Bogotá Colombia.

Pérez, A. (2004). Particularidades del Método Interpretativo Crítico. Madrid: Morata.

Rusque, A. (2001) De la diversidad a la unidad en la investigación cualitativa. Ediciones FACES/UCVEditorial Vadell Hermanos. Venezuela

Sandín, M (2003) Investigación cualitativa en educación. Fundamentos y Tradiciones. Madrid

Sorman, M (2005), Epistemología y Metodología Elementos Para Una Teoría de la Investigación Científica. Universidad de Buenos aires

Tedesco, C. (1999). La Investigación Educativa en América Latina, Revisión y Estudios Previos. México: Trillas.

Téllez, G. (2009). La Investigación Educativa en el marco de los cambios paradigmáticos. Congreso nacional de Investigación. Barranquilla, Colombia.

Taylor, S. y Bogdan, R. (1990).Introducción a los Métodos Cualitativos de Investigación. Barcelona: Paidos.

UNESCO (1998). La declaración mundial sobre educación superior, en el siglo XXI. UNESCO. París. Francia.

Zaa, J. (2008). La Investigación Científica. Entre la Imaginación Creadora y el reduccionismo del Lenguaje. Ponencia Internacional de Política Ética y Filosofía. Congreso de Investigación La Grita Estado Táchira. Venezuela. 